Modern Physics Letters A

(C) World Scientific Publishing Company

\title{
ALTERNATIVES TO THE INFLATIONARY PARADIGM OF STRUCTURE FORMATION
}

\author{
ROBERT H. BRANDENBERGER \\ Physics Department, McGill University, 3600 University Str., \\ Montreal, Quebec, H3A 2T8, Canada \\ and \\ Institute of High Energy Physics, Chinese Academy of Sciences, P.O. Box 918-4 \\ Beijing 100049, P.R. China \\ rhb@physics.mcgill.ca
}

Received (Day Month Year)

Revised (Day Month Year)

\begin{abstract}
The inflationary paradigm, although very successful phenomenologically, suffers from several conceptual problems which motivate the search for alternative scenarios of early universe cosmology. Here, two possible alternatives will be reviewed. - "string gas cosmology" and the "matter bounce". Their successes and problems will be pointed out.

Keywords: Keyword1; keyword2; keyword3.
\end{abstract}

PACS Nos.: include PACS Nos.

\section{Introduction}

The inflationary paradigm of modern cosmology is based on the assumption that there was a time period starting at time $t_{i}$ and ending at time $t_{R}$ during which the universe undergoes accelerated expansion 1. Inflation explains the overall homogeneity and isotropy of the universe. Assuming that the universe was flat enough and large enough initially to reach the phase of accelerated expansion, inflation can explain the large size and entropy of the current universe, and its spatial flatness. Most importantly, however, the inflationary paradigm gave rise to a predictive theory of the origin of the primordial cosmological perturbations ${ }^{2}$ (see also [3, 4]).

Figure 1 is a space-time sketch of inflationary cosmology. The vertical axis is time, the horizontal axis indicates physical length. The Hubble radius $H^{-1}$ is the key length scale in cosmology. It separates small scales on which matter forces dominate from large scales where matter is frozen in and gravity dominates. The accelerated expansion of space which inflation provides results in wavelengths which start out at early time with wavelength smaller than $H^{-1}$ being stretched to become super-Hubble. Thus it is possible to have a causal generation mechanism for fluctuations. Since any classical matter present at the beginning of inflation red-shifts, it 
is reasonable to assume 2 that perturbations emerge as quantum vacuum fluctuations. This mechanism produces an almost scale-invariant spectrum of cosmological perturbations. Thus, the first key requirement on a theory of structure formation which can explain current data is satisfied. The perturbations are frozen in at Hubble radius crossing and propagate on super-Hubble scales without being sourced by other matter effects. This "coasting" of the fluctuations on super-Hubble scales is the second crucial requirement in order to obtain the characteristic oscillations in the angular power spectrum of the cosmic microwave anisotropies.

Current models of inflation are based on using general relativity as the theory of space-time, and making use of a scalar matter field to generate the phase of accelerated expansion (see, however, [5] for the initial model in which it is correction terms to the gravitational action which lead to inflation). More specifically, it is required to have a phase during which the energy-momentum tensor of matter is dominated by the potential energy of the scalar field.

The reliance of inflationary cosmology on scalar fields satisfying stringent constraints constitutes a first problem for inflation. In order to make sure that the scalar field rolls sufficiently slowly for a sufficiently long time, its potential must obey various "slow-roll" conditions. In addition, initial conditions for the scalar field must be appropriately chosen, and the scalar field must be correctly coupled to regular matter to enable energy transfer from it to regular matter. Once these conditions are satisfied, the slope of the potential has to be fine-tuned in order for the amplitude of the induced density fluctuations not to exceed the observational bounds 6 .

A further problem for the inflationary scenario (at least when realized with scalar matter fields) is the presence of an initial singularity 7 . Thus, inflationary cosmology cannot provide a complete description of the early universe.

A more serious problem for inflation is the trans-Planckian problem for cosmological fluctuations $8 \mid 9$. The same mechanism which inflates the wavelength of fluctuations such that they begin on sub-Hubble scales leads to the conclusion that, provided inflation lasted just a few e-foldings of expansion more than what is required for the scenario to solve the homogeneity and flatness problems, then the physical wavelength of all scales which are being observed today is less than the Planck length at the beginning of inflation. Thus, as illustrated in Figure 1, the fluctuations emerge from a "zone of ignorance" where we have no right to use the Einstein action. It is easy to construct toy models of trans-Planckian physics which lead to large corrections in the predictions of inflationary cosmology 9 .

Along the same lines, the applicability of the Einstein effective field theory equations for the background space-time can be questioned at energy scales relevant for inflation (about $10^{16} \mathrm{GeV}$ in single field slow-roll inflation models with simple potentials). All approaches to quantum gravity tell us that terms in the action other than the Einstein term will dominate at scales close to the quantum gravity scale. The quantum gravity scale is close to the energy scale of inflation, and thus us- 


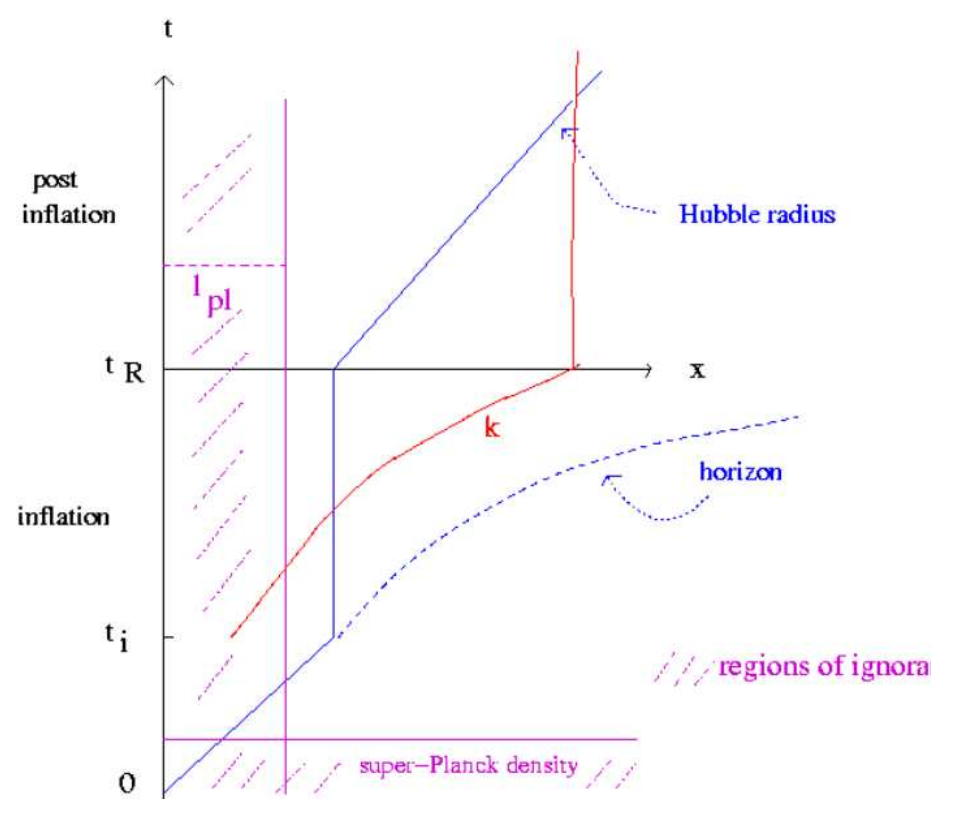

Fig. 1. Space-time sketch of inflationary cosmology. The vertical axis is time, the horizontal one is physical distance. The length scales shown are the Hubble radius (vertical during the inflationary phase - assumed here to be exponential expansion), the horizon (dashed line) and the physical wavelength corresponding to a fixed co-moving scale (the curve indicated by the label $k$ ). This figure also llustrates the trans-Planckian problem for fluctuations in inflationary cosmology: If the period of inflation is sufficiently long, then at the beginning of the inflationary phase the physical wavelength of all scales which are being observed today is smaller than the Planck length (the vertical curve indicated by $l_{p l}$ ). Finally, there is a high density zone of ignorance which may extend to densities relevant for inflation.

ing the Einstein action at these energies may be giving us the wrong result. These conceptual problems of inflation are discussed in more detail in [10].

In light of the conceptual problems outlined above it is important to study pos- 
sible alternative scenarios for early universe cosmology. In the following we will introduce two alternative scenarios, "string gas cosmology" and the "matter bounce". Both of these scenarios are immune to the trans-Planckian problem for cosmological fluctuations. Both a designed to yield non-singular cosmologies. Both make predictions with which they can be distinguished from inflationary cosmology, but both also have their set of conceptual problems.

\section{String Gas Cosmology}

String gas cosmology 11 (see also 12 and 13, 14 for reviews with extensive lists of references) is a toy model of the very early universe which makes use of key new degrees of freedom and symmetries of string theory (which are not present in quantum particle theories). It is based on coupling a gas of closed string matter to a background space-time geometry.

We assume that all spatial sections are compact. For simplicity, we assume that they are toroidal, with $R$ denoting the radius of the torus). In this case, the degrees of freedom of closed strings include, in addition to the momentum modes whose energies are quantized in units of $1 / R$, string winding modes whose energies are quantized in units of $R$, and string oscillatory modes (whose energies are independent of $R$ ). For comparison, the only degrees of freedom which point particles have are momentum modes.

The number of oscillatory modes of a closed string increases exponentially with energy. Hence $\frac{15}{15}$ there is a maximal temperature of a gas of strings, the "Hagedorn temperature" $T_{H}$. The presence of string winding modes leads to a symmetry of the string mass spectrum under the transformation $R \rightarrow 1 / R$ (in string units). Under this transition, momentum and winding modes are exchanged. This is a special case of the more general T-duality symmetry.

Let us imagine a contracting box of strings in thermal equilibrium. Initially, almost all of the energy is in the momentum modes which are light at large $R$. Thus, the temperature $T$ will rise as $R$ decreases as in standard particle cosmology. However, as $T$ approaches $T_{H}$, the energy will gradually drift from the momentum modes to the oscillatory modes. Once $R$ decreases below 1 , the energy will move to the winding modes, the modes which are light at small values of $R$, and the temperature will get smaller as $R$ decreases further. The temperature-radius evolution is sketched in Figure 2 11.

In order to determine the space-time dynamics of string gas cosmology, we need to know how $R$ evolves as a function of time $t$. There are two possibilities. The first, the one we adopt here, is that the universe begins close to the Hagedorn temperature. This configuration is a metastable fixed point (a fixed point because of the T-duality symmetry, metastable because the decay of string winding modes into loops will lead to a dynamical breaking of this symmetry). The resulting time evolution of the scale factor is sketched in Figure 3. Another possibility is that $R$ begins much smaller than string scale and monotonically increases. This will give 


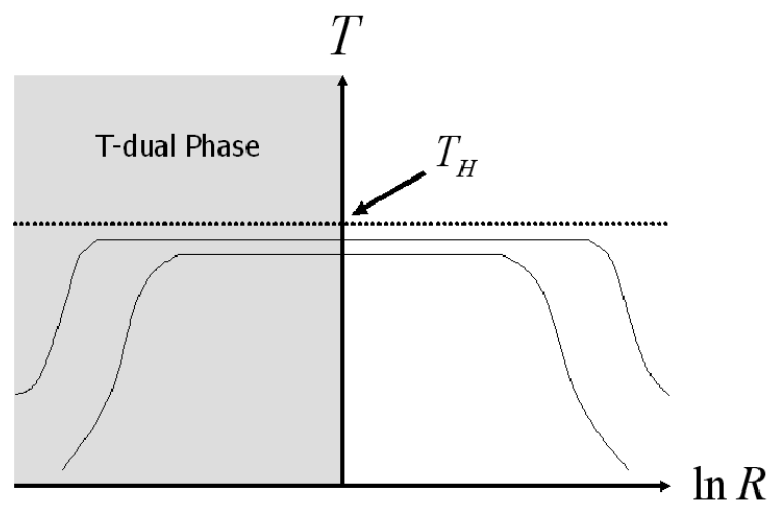

Fig. 2. Temperature-size relation in string gas cosmology. $R$ is the radius of the torus, $T$ the temperature. The temperature approaches but never reaches the limiting temperature $T_{H}$.

rise to a cosmology in which $T(R)$ evolves as in a bouncing universe.

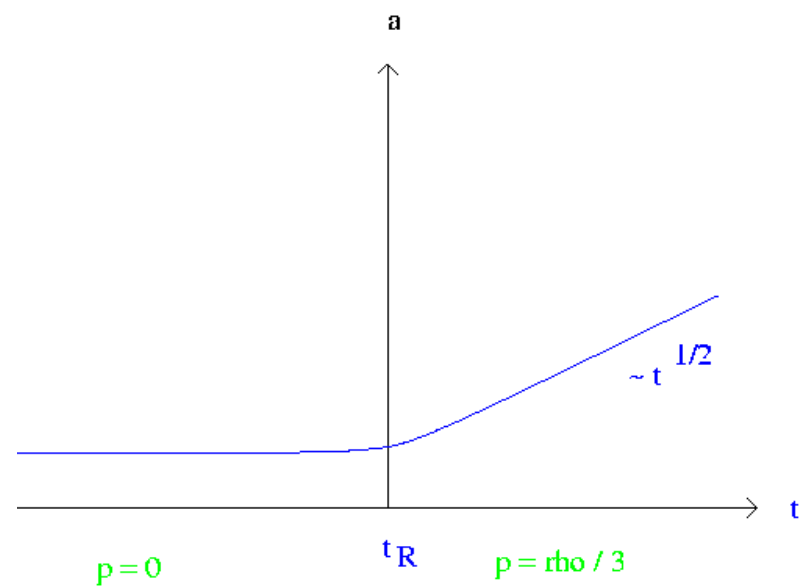

Fig. 3. The time dependence of the scale factor $a$ in string gas cosmology.

As is apparent from the above, there is no temperature singularity in string gas cosmology 11. In addition, one can argue 11 that the annihilation of winding modes which is required in order for space to expand is possible in at most three spatial dimensions, and that string gas cosmology thus provides an explanation for the fact that we see only three large spatial dimensions (concerns about this argument have been raised in [16, 17). The interaction of string winding and momentum modes leads to a dynamical stabilization of all of the size ${ }^{18 / 19}$ and shape ${ }^{20}$ moduli of the extra dimensions, without the need for any additional inputs. This progress has been 
reviewed in detail in 21. The only modulus field which must be stabilized "by hand" is the dilaton. This can be done ${ }^{22}$, however, using non-perturbative techniques of the same type which are used for modulus stabilization in string inflation model building.

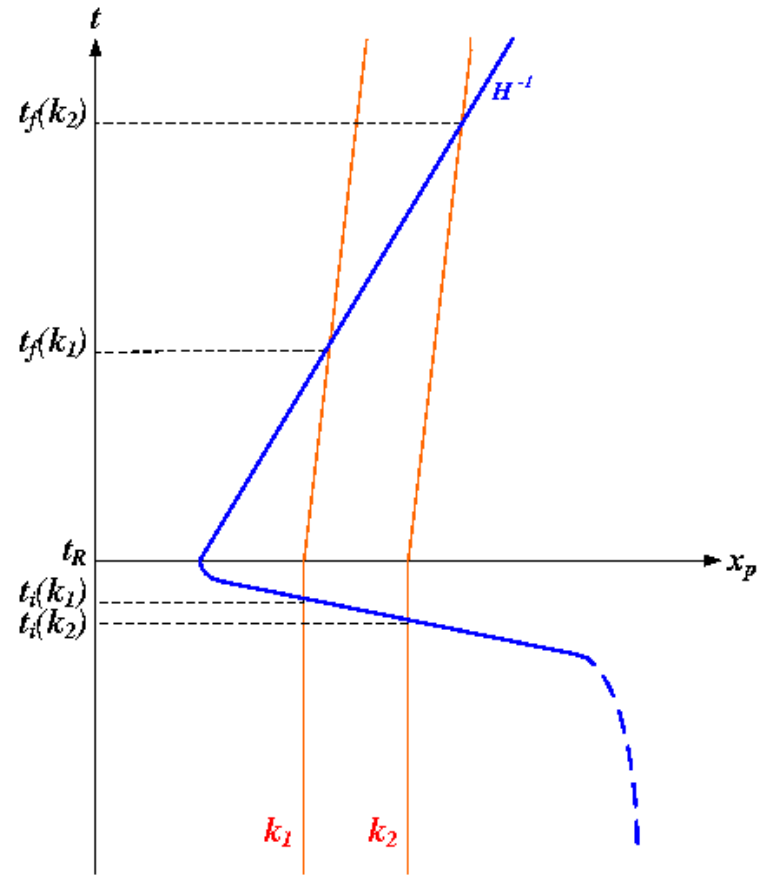

Fig. 4. Space-time sketch in string gas cosmology. The vertical axis is time, the horizontal axis physical distance, as in Figure 1. Two co-moving scales are shown, and the time when a scale $k$ exits the Hubble radius at the end of the Hagedorn phase is denoted $t_{i}(k)$. The time $t_{R}$ denotes the end of the Hagedorn phase when the winding modes have annihilated into string radiation.

Let us now turn to the issue of fluctuations in string gas cosmology. Figure 4 presents a space-time sketch of string gas cosmology. Since the Hagedorn phase is 
quasi-static, the Hubble radius tends to infinity as we get deep into the Hagedorn phase. As is clear from the sketch, fixed co-moving scales which are subject of observational cosmology today originate inside the Hubble radius. If the energy density at the transition time $t_{R}$ corresponding to the end of the Hagedorn phase is of the order of $10^{16} \mathrm{GeV}$, then the physical wavelength during the Hagedorn phase corresponding to the present Hubble radius is about $1 \mathrm{~mm}$, i.e. a scale in the far infrared, well removed from the trans-Planckian zone of ignorance.

Unlike in inflationary cosmology, where the fluctuations are of quantum vacuum nature because any classical matter is being red-shifted at an accelerated rate, in string gas cosmology the dominant fluctuations are thermal fluctuations of the dominant matter, namely thermal string fluctuations. As was recently realized ${ }^{23}$, thermal string gas fluctuations in the Hagedorn phase yield a scale-invariant spectrum of cosmological perturbations at late times. Thus, string gas cosmology provides an alternative to the theory of cosmological inflation in terms of providing a causal theory of structure formation.

The procedure for computing the spectrum of cosmological perturbations and gravitational waves is as follows: first, we compute the matter fluctuations on subHubble scales during the Hagedorn phase making use of results from closed string thermodynamics ${ }^{24}$. Next, for each co-moving scale $k$, we compute the resulting metric fluctuations at the time $t_{H}(k)$ when the mode exits the Hubble radius, making use of the Einstein perturbation constraint equations. Finally, the metric fluctuations evolve freely on super-Hubble scales until they re-enter the Hubble radius at late times.

We start by writing the metric including linear cosmological perturbations and gravitational waves in the following gauge-fixed form (see e.g. ${ }^{25}$ for an in-depth review of the theory of cosmological perturbations, and ${ }^{26}$ for a brief overview):

$$
d s^{2}=a^{2}(\eta)\left((1+2 \Phi) d \eta^{2}-\left[(1-2 \Phi) \delta_{i j}+h_{i j}\right] d x^{i} d x^{j}\right) .
$$

Here, $\eta$ is conformal time, $\Phi(x, \eta)$ describes the cosmological perturbations (assuming the absence of anisotropic stress), and the transverse traceless tensor $h_{i j}$ represents the gravitational waves.

Inserting into the perturbed Einstein equations yields

$$
\left\langle|\Phi(k)|^{2}\right\rangle=16 \pi^{2} G^{2} k^{-4}\left\langle\delta T^{0}{ }_{0}(k) \delta T^{0}{ }_{0}(k)\right\rangle,
$$

and

$$
\left\langle|h(k)|^{2}\right\rangle=16 \pi^{2} G^{2} k^{-4}\left\langle\delta T^{i}{ }_{j}(k) \delta T^{i}{ }_{j}(k)\right\rangle(i \neq j) .
$$

We thus conclude that the cosmological perturbations are given by the energy density fluctuations whereas the gravitational waves are determined by the off-diagonal stress fluctuations.

For thermal matter, the energy density fluctuations are given by the specific heat capacity $C_{V}$ (where $V$ indicates the volume). In a box of radius $R$, one has

$$
\left\langle\delta \rho^{2}\right\rangle=\frac{T^{2}}{R^{6}} C_{V} .
$$


For a gas of closed strings on a space with stable winding modes, the specific heat capacity has the holographic form

$$
C_{V} \approx 2 \frac{R^{2} / \ell_{s}^{3}}{T\left(1-T / T_{H}\right)}
$$

at temperatures close to the Hagedorn value.

Combining these results, we obtain the following power spectrum of cosmological perturbations

$$
\begin{aligned}
P_{\Phi}(k) & \equiv k^{3}|\Phi(k)|^{2}=8 G^{2} k^{-1}<|\delta \rho(k)|^{2}> \\
& =8 G^{2} k^{2}<(\delta M)^{2}>_{R}=8 G^{2} k^{-4}<(\delta \rho)^{2}>_{R} \\
& =8 G^{2} \frac{T}{\ell_{s}^{3}} \frac{1}{1-T / T_{H}}
\end{aligned}
$$

where for each value of $k$, the temperature $T$ is evaluated at the time $t_{H}(k)$ of Hubble radius crossing, and $\ell_{s}$ denotes the string length.

Since the temperature is approximately constant during the Hagedorn phase, this power spectrum is scale-invariant. Taking into account the fact that larger scales exit the Hubble radius at slightly higher temperatures, one obtains a slight red tilt of the spectrum (more power on longer wavelengths). These are identical to the results obtained in inflationary models.

The predictions of string gas cosmology can be differentiated from those of the inflationary scenario by considering the spectrum of gravitational waves 27. By an analysis similar to the one above, we find the following power spectrum of gravitational waves:

$$
\begin{aligned}
P_{h}(k) & \equiv k^{3}|h(k)|^{2}=16 \pi^{2} G^{2} k^{-1}<\left|T_{i j}(k)\right|^{2}> \\
& =16 \pi^{2} G^{2} k^{-4}<\left|T_{i j}(R)\right|^{2}>\sim 16 \pi^{2} G^{2} \frac{T}{\ell_{s}^{3}}\left(1-T / T_{H}\right) .
\end{aligned}
$$

The key ingredient from string thermodynamics is $28 \mid 29$

$$
<\left|T_{i j}(R)\right|^{2}>\sim \frac{T}{l_{s}^{3} R^{4}}\left(1-T / T_{H}\right) .
$$

This spectrum is once again scale invariant, but it has a slight blue tilt, whereas the gravitational wave spectrum in inflationary models always has a slight red tilt. The origin of the slight blue tilt is easy to understand. Long wavelengths exit the Hubble radius deeper in the Hagedorn phase when the pressure is closer to zero and hence the off-diagonal pressure fluctuations are smaller 27 .

Let us summarize the requirements which have to be satisfied in order for the string gas cosmology structure formation scenario to work. Firstly, there needs to be a quasi-static Hagedorn phase. Based on the string symmetries we have discussed, we expect such a phase to exist. Obviously, this phase cannot be described using Einstein gravity since Einstein gravity does not obey the T-duality symmetry of string theory. Dilaton gravity is not satisfactory, either ${ }^{30}$, since it does not include 


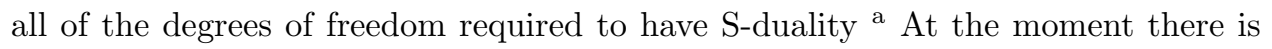
no satisfactory effective field theory description of the Hagedorn phase of string gas cosmology (for some attempts in this direction see [33]).

The second key requirement for our scenario is that the specific heat capacity has the holographic scaling $C_{V}(R) \sim R^{2}$. Finally, on infrared scales, the perturbed background equations of motion must reduce to those in Einstein gravity. All these criteria can be satisfied in the non-singular bouncing cosmology of [34, 35].

\section{Matter Bounce}

Another way to obtain a scale-invariant spectrum of cosmological perturbations is from quantum vacuum fluctuations which exit the Hubble radius in a contracting matter-dominated universe $36|37| 38 \mid 39$. Provided that this phase of contraction can be connected smoothly to the expanding phase of standard cosmology, a paradigm alternative to inflation for generating the observed structure will result.

There are various ways to obtain a non-singular bounce. One way is by introducing new matter fields with opposite sign kinetic terms (see e.g. 40, 41, 38, 42]). A second way is by making use of specifically chosen higher derivative terms in the gravitational action. A special choice of the gravitational action which leads to a non-singular bounce and at the same time is ghost-free is given in 34 and discussed in more detail in 35. A new realization of the first approach is the "Lee-Wick bounce" 43, a bouncing cosmology obtained from the scalar sector of the Lee-Wick Standard Model ${ }^{44}$, an alternative to supersymmetry for solving the hierarchy problem of the Standard Model of particle physics.

The scalar sector of the Lee-Wick Standard Model is given by the following Lagrangian:

$$
\mathcal{L}=\frac{1}{2} \partial_{\mu} \phi \partial^{\mu} \phi-\frac{1}{2} \partial_{\mu} \tilde{\phi} \partial^{\mu} \tilde{\phi}+\frac{1}{2} M^{2} \tilde{\phi}^{2}-\frac{1}{2} m^{2} \phi^{2}-\frac{\lambda}{4}(\phi-\tilde{\phi})^{4},
$$

where $\phi$ is the Higgs and $\tilde{\phi}$ is its Lee-Wick partner. The mass scale $M$ is the scale of the new physics. We expect it to be much larger than the masses of the Standard Model particles.

The above Lagrangian gives rise to a non-singular bounce in the following way 43 : We begin the evolution in a contracting phase with both matter fields oscillating, and the energy dominated by $\phi$. Thus, the amplitude of $\phi$ is much larger than that of $\tilde{\phi}$. The amplitudes of oscillation of both fields scale as $a^{-3 / 2}$. Eventually, the amplitude of $\phi$ reaches the value $m_{p l}$ (Planck mass), and $\phi$ stops to oscillate. Its amplitude now increases only slowly, whereas the amplitude of $\tilde{\phi}$ continues to increase as $a(t)^{-3 / 2}$. Thus, the (negative) energy density in $\tilde{\phi}$ rapidly catches up to

a Note that the objections to string gas cosmology of [31,32] are based on computations done in the context of dilaton gravity and do not apply to the setup described here. 
the (positive) energy density in $\phi$. From the Friedmann equations

$$
\begin{aligned}
H^{2} & =\frac{8 \pi G}{3}\left[\frac{1}{2} \dot{\phi}^{2}-\frac{1}{2} \dot{\tilde{\phi}}^{2}+\frac{1}{2} m^{2} \phi^{2}-\frac{1}{2} M^{2} \tilde{\phi}^{2}+\frac{\lambda}{4}(\phi-\tilde{\phi})^{4}\right], \\
\dot{H} & =-4 \pi G\left(\dot{\phi}^{2}-\dot{\tilde{\phi}}^{2}\right)
\end{aligned}
$$

it then follows that $H=0$ and $\dot{H}>0$ (the latter since $\tilde{\phi}$ has large kinetic energy whereas $\phi$ only has small kinetic energy). Thus, a non-singular bounce is obtained quite naturally. After the bounce, the universe soon enters a phase of matter-dominated expansion.

Figure 5 is a sketch of the space-time which results in the matter bounce scenario. The vertical axis is time, the horizontal axis physical distance. The length scales shown are the Hubble radius as well as the wavelength corresponding to a fixed co-moving scale $k$.

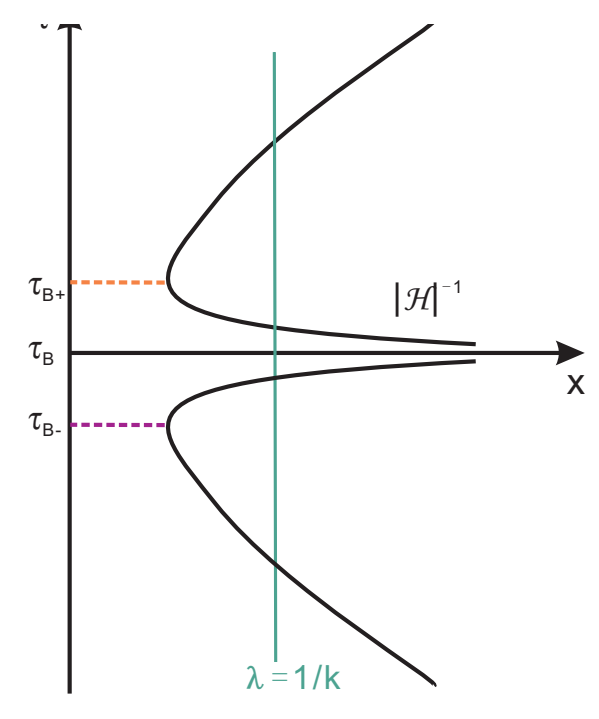

Fig. 5. Space-time sketch in the matter bounce scenario. The vertical axis is conformal time $\eta$, the horizontal axis denotes a co-moving space coordinate. Also, $\mathcal{H}^{-1}$ denotes the co-moving Hubble radius.

Let us now briefly recall how initial quantum vacuum fluctuations in the contracting matter phase evolve into a scale-invariant spectrum of cosmological perturbations at late times. It is easiest to work in terms of the variable $\zeta$, the curvature fluctuation in co-moving coordinates. The variable $\zeta$ is closely related to the SasakiMukhanov variable $45 \mid 46] v=z \zeta$ in terms of which the action for cosmological perturbations has canonical kinetic term. The equation of motion for the Fourier mode 
$v_{k}$ of $v$ is

$$
v_{k}^{\prime \prime}+\left(k^{2}-\frac{z^{\prime \prime}}{z}\right) v_{k}=0,
$$

where the prime indicates a derivative with respect to conformal time. If the equation of state of the background is time-independent, then $z \sim a$ and hence the negative square mass term in 12 is $H^{2}$. Thus, on length scales smaller than the Hubble radius, the solutions of $(12)$ are oscillating, whereas on larger scales they are frozen in as standing waves, and their amplitude depends on the time evolution of $z$.

On super-Hubble scales, the equation of motion 12 for $v_{k}$ in a universe which is contracting or expanding as a power $p$ of physical time $t$, i.e.

$$
a(t) \sim t^{p}
$$

becomes

$$
v_{k}^{\prime \prime}=\frac{p(2 p-1)}{(p-1)^{2}} \eta^{-2} v_{k}
$$

which has solutions

$$
v(\eta) \sim \eta^{\alpha}
$$

with

$$
\alpha=\frac{1}{2} \pm \nu, \quad \nu=\frac{1}{2} \frac{1-3 p}{1-p} .
$$

In the case of a matter-dominated contraction we have $\nu=-3 / 2$ and hence

$$
v_{k}(\eta)=c_{1} \eta^{2}+c_{2} \eta^{-1}
$$

where $c_{1}$ and $c_{2}$ are again constants. The $c_{1}$ mode is the mode for which $\zeta$ is constant on super-Hubble scales. However, in a contracting universe it is the $c_{2}$ mode which dominates and leads to a scale-invariant spectrum $36|37| 38$.

$$
\left.P_{\zeta}(k, \eta) \sim k^{3}\left|v_{k}(\eta)^{2} a^{-2}(\eta) \sim k^{3}\right| v_{k}\left(\eta_{H}(k)\right)\right|^{2}\left(\frac{\eta_{H}(k)}{\eta}\right)^{2} \sim k^{3-1-2} \sim \text { const },
$$

making us of the scaling of the dominant mode of $v_{k}$, the Hubble radius crossing condition $\eta_{H}(k) \sim k^{-1}$, and the vacuum spectrum $v_{k} \sim k^{-1 / 2}$ at Hubble radius crossing.

The above analysis shows that on scales larger than the Hubble radius, a scaleinvariant spectrum of fluctuations in obtained in the contracting phase. The equation of motion for $\zeta$ has a singularity at the bounce which is connected with the fact that the co-moving gauge becomes ill-defined at that point. What was done in [43] is therefore to carefully study the evolution of $\Phi$ through the bounce. The analysis was done numerically without any approximations, and analytically by solving the equation of motion for $\Phi$ approximately in the matter-dominated contracting phase, in the bounce phase, where the Hubble constant can be modeled as $H(t)=\alpha t, t=0$ 
denoting the bounce time, and finally in the matter-dominated phase of expansion, and making use of the matching conditions for fluctuations $47 \mid 48$ at the two phase transitions. Note that the use of these matching conditions is justified since at each transition surface the background also satisfies the background matching conditions.

Like string gas cosmology, the matter bounce scenario can solve the singularity problem of inflation. Again like in string gas cosmology, the wavelength of fluctuations of current interest in observational cosmology is in the far infrared at all times, and thus the trans-Planckian problem for fluctuations does not arise.

Matter bounce scenarios are plagued by the problem of ghosts 49 . This is a particularly serious problem in models like the Lee-Wick bounce where new matter fields with phantom behavior are introduced (whereas the higher derivative gravity model of 34 is free of ghosts). As long as the energy density remains smaller than those corresponding to the phantom field mass scale $M$ (as it does in the case of the Lee-Wick bounce), the model may be considered as a low-energy effective field theory emerging from a fundamental theory free of ghosts.

A matter bounce scenario is unstable to the presence of radiation and, more importantly, of anisotropies This is a further problem which must be addressed.

\section{Discussion and Conclusions}

The main message of this talk is that there are scenarios other than inflation which can successfully connect fundamental physics with observations. The focus of this lecture has been on string gas cosmology and on the matter bounce scenario. This is, however, by no means a complete list of alternatives. Pre-Big-Bang cosmology and the Ekpyrotic/Cyclic scenarios are other alternatives, which for lack of space cannot be described here.

\section{Acknowledgments}

I wish to thank the organizers of CosPA08 for the invitation to speak and for their wonderful hospitality in Pohang. I wish to thank Yifu Cai, Taotao Qiu and Xinmin Zhang for introducing me to the Lee-Wick model and for collaborating on [43, and Yifu Cai, Sugumi Kanno and Subodh Patil for allowing me to use a figure they each drew. I am grateful to Professor X. Zhang and the Theory Division of the Institute of High Energy Physics (IHEP) for their hospitality and financial support. My research is also supported by an NSERC Discovery Grant and by the Canada Research Chairs Program.

\section{References}

1. A. H. Guth, "The Inflationary Universe: A Possible Solution To The Horizon And Flatness Problems," Phys. Rev. D 23, 347 (1981).

2. V. F. Mukhanov and G. V. Chibisov, "Quantum Fluctuation And 'Nonsingular' Universe. (In Russian)," JETP Lett. 33, 532 (1981) [Pisma Zh. Eksp. Teor. Fiz. 33, 549 (1981)]. 
3. W. Press, "Spontaneous production of the Zel'dovich spectrum of cosmological fluctuations", Phys. Scr. 21, 702 (1980).

4. K. Sato, "First Order Phase Transition Of A Vacuum And Expansion Of The Universe," Mon. Not. Roy. Astron. Soc. 195, 467 (1981).

5. A. A. Starobinsky, "A New Type Of Isotropic Cosmological Models Without Singularity," Phys. Lett. B 91, 99 (1980).

6. F. C. Adams, K. Freese and A. H. Guth, "Constraints on the scalar field potential in inflationary models," Phys. Rev. D 43, 965 (1991).

7. A. Borde and A. Vilenkin, "Eternal inflation and the initial singularity," Phys. Rev. Lett. 72, 3305 (1994) arXiv:gr-qc/9312022.

8. R. H. Brandenberger, "Inflationary cosmology: Progress and problems," arXiv:hep$\mathrm{ph} / 9910410$.

9. J. Martin and R. H. Brandenberger, "The trans-Planckian problem of inflationary cosmology," Phys. Rev. D 63, 123501 (2001) arXiv:hep-th/0005209.

10. R. H. Brandenberger, "Looking beyond inflationary cosmology," arXiv:hepth/0509076

11. R. H. Brandenberger and C. Vafa, "Superstrings In The Early Universe," Nucl. Phys. B 316, 391 (1989).

12. J. Kripfganz and H. Perlt, "Cosmological Impact Of Winding Strings," Class. Quant. Grav. 5, 453 (1988).

13. T. Battefeld and S. Watson, "String gas cosmology," Rev. Mod. Phys. 78, 435 (2006) arXiv:hep-th/0510022.

14. R. H. Brandenberger, "String Gas Cosmology," arXiv:0808.0746 [hep-th].

15. R. Hagedorn, "Statistical Thermodynamics Of Strong Interactions At High-Energies," Nuovo Cim. Suppl. 3, 147 (1965).

16. R. Easther, B. R. Greene, M. G. Jackson and D. Kabat, "String windings in the early universe," JCAP 0502, 009 (2005) arXiv:hep-th/0409121.

17. R. Danos, A. R. Frey and A. Mazumdar, "Interaction rates in string gas cosmology," Phys. Rev. D 70, 106010 (2004) arXiv:hep-th/0409162.

18. S. P. Patil and R. H. Brandenberger, "The cosmology of massless string modes," arXiv:hep-th/0502069

19. S. Watson and R. Brandenberger, "Stabilization of extra dimensions at tree level," JCAP 0311, 008 (2003) arXiv:hep-th/0307044.

20. R. Brandenberger, Y. K. Cheung and S. Watson, "Moduli stabilization with string gases and fluxes," JHEP 0605, 025 (2006) arXiv:hep-th/0501032.

21. R. H. Brandenberger, "Moduli stabilization in string gas cosmology," Prog. Theor. Phys. Suppl. 163, 358 (2006) arXiv:hep-th/0509159.

22. R. J. Danos, A. R. Frey and R. H. Brandenberger, "Stabilizing moduli with thermal matter and nonperturbative effects," Phys. Rev. D 77, 126009 (2008) arXiv:0802.1557 [hep-th]].

23. A. Nayeri, R. H. Brandenberger and C. Vafa, "Producing a scale-invariant spectrum of perturbations in a Hagedorn phase of string cosmology," Phys. Rev. Lett. 97, 021302 (2006) arXiv:hep-th/0511140.

24. N. Deo, S. Jain, O. Narayan and C. I. Tan, "The Effect of topology on the thermodynamic limit for a string gas," Phys. Rev. D 45, 3641 (1992).

25. V. F. Mukhanov, H. A. Feldman and R. H. Brandenberger, "Theory of cosmological perturbations. Part 1. Classical perturbations. Part 2. Quantum theory of perturbations. Part 3. Extensions," Phys. Rept. 215, 203 (1992).

26. R. H. Brandenberger, "Lectures on the theory of cosmological perturbations," Lect. Notes Phys. 646, 127 (2004) arXiv:hep-th/0306071. 
27. R. H. Brandenberger, A. Nayeri, S. P. Patil and C. Vafa, "Tensor modes from a primordial Hagedorn phase of string cosmology," Phys. Rev. Lett. 98, 231302 (2007) arXiv:hep-th/0604126.

28. A. Nayeri, "Inflation free, stringy generation of scale-invariant cosmological fluctuations in $\mathrm{D}=3+1$ dimensions," arXiv:hep-th/0607073.

29. R. H. Brandenberger, A. Nayeri, S. P. Patil and C. Vafa, "String gas cosmology and structure formation," Int. J. Mod. Phys. A 22, 3621 (2007) arXiv:hep-th/0608121.

30. R. H. Brandenberger et al., "More on the Spectrum of Perturbations in String Gas Cosmology," JCAP 0611, 009 (2006) arXiv:hep-th/0608186.

31. N. Kaloper, L. Kofman, A. Linde and V. Mukhanov, "On the new string theory inspired mechanism of generation of cosmological perturbations," JCAP 0610, 006 (2006) arXiv:hep-th/0608200.

32. N. Kaloper and S. Watson, "Geometric Precipices in String Cosmology," Phys. Rev. D 77, 066002 (2008) arXiv:0712.1820 [hep-th]].

33. R. H. Brandenberger, A. R. Frey and S. Kanno, "Towards A Nonsingular Tachyonic Big Crunch," Phys. Rev. D 76, 063502 (2007) arXiv:0705.3265 [hep-th]].

34. T. Biswas, A. Mazumdar and W. Siegel, "Bouncing universes in string-inspired gravity," JCAP 0603, 009 (2006) arXiv:hep-th/0508194;

35. T. Biswas, R. Brandenberger, A. Mazumdar and W. Siegel, "Non-perturbative gravity, Hagedorn bounce and CMB," arXiv:hep-th/0610274.

36. D. Wands, "Duality invariance of cosmological perturbation spectra," Phys. Rev. D 60, 023507 (1999) arXiv:gr-qc/9809062.

37. F. Finelli and R. Brandenberger, "On the generation of a scale-invariant spectrum of adiabatic fluctuations in cosmological models with a contracting phase," Phys. Rev. D 65, 103522 (2002) arXiv:hep-th/0112249.

38. L. E. Allen and D. Wands, "Cosmological perturbations through a simple bounce," Phys. Rev. D 70, 063515 (2004) arXiv:astro-ph/0404441.

39. P. Peter and N. Pinto-Neto, "Cosmology without inflation," Phys. Rev. D 78, 063506 (2008) arXiv:0809.2022 [gr-qc]].

40. P. Peter and N. Pinto-Neto, "Primordial perturbations in a non singular bouncing universe model," Phys. Rev. D 66, 063509 (2002) arXiv:hep-th/0203013.

41. F. Finelli, "Study of a class of four dimensional nonsingular cosmological bounces," JCAP 0310, 011 (2003) arXiv:hep-th/0307068.

42. Y. F. Cai, T. Qiu, Y. S. Piao, M. Li and X. Zhang, "Bouncing Universe with Quintom Matter," JHEP 0710, 071 (2007) arXiv:0704.1090 [gr-qc]].

43. Y. F. Cai, T. Qiu, R. Brandenberger and X. Zhang, "A Nonsingular Cosmology with a Scale-Invariant Spectrum of Cosmological Perturbations from Lee-Wick Theory," arXiv:0810.4677 [hep-th].

44. B. Grinstein, D. O'Connell and M. B. Wise, "The Lee-Wick standard model," Phys. Rev. D 77, 025012 (2008) arXiv:0704.1845 [hep-ph]].

45. M. Sasaki, "Large Scale Quantum Fluctuations in the Inflationary Universe," Prog. Theor. Phys. 76, 1036 (1986).

46. V. F. Mukhanov, "Quantum Theory of Gauge Invariant Cosmological Perturbations," Sov. Phys. JETP 67, 1297 (1988) [Zh. Eksp. Teor. Fiz. 94N7, 1 (1988)].

47. J. C. Hwang and E. T. Vishniac, "Gauge-invariant joining conditions for cosmological perturbations," Astrophys. J. 382, 363 (1991).

48. N. Deruelle and V. F. Mukhanov, "On matching conditions for cosmological perturbations," Phys. Rev. D 52, 5549 (1995) arXiv:gr-qc/9503050.

49. J. M. Cline, S. Jeon and G. D. Moore, "The phantom menaced: Constraints on lowenergy effective ghosts," Phys. Rev. D 70, 043543 (2004) arXiv:hep-ph/0311312. 